\title{
Interval Type-2 Fuzzy PD Tracking Control of Flexible-Joint Robots
}

\author{
Majid Moradi Zirkohi, Samsam Izadpanah \\ Department of Electrical Engineering, Behbahan Khatam Alanbia University of Technology, Behbahan, Iran \\ Email: Izadpanah@bkatu.ac.ir, Moradi@bkatu.ac.ir
}

How to cite this paper: Zirkohi, M.M. and Izadpanah, S. (2017) Interval Type-2 Fuzzy PD Tracking Control of Flexible-Joint Robots. Journal of Software Engineering and Applications, 10, 854-872. https://doi.org/10.4236/jsea.2017.1011048

Received: April 22, 2017

Accepted: October 28, 2017

Published: October 31, 2017

Copyright $\odot 2017$ by authors and Scientific Research Publishing Inc. This work is licensed under the Creative Commons Attribution International License (CC BY 4.0).

http://creativecommons.org/licenses/by/4.0/

(c) (i) Open Access

\begin{abstract}
This paper develops a novel interval type-2 fuzzy Proportional-Derivative (PD) control scheme for electrically driven flexible-joint robots using the direct method of Lyapunov. The controller has a simple design in a decentralized structure. Compared to the previous controllers reported for the flexible-joint robots which use two control loops, it has a simpler structure using only one control loop. It guarantees stability and provides a good tracking performance. The controller considers the whole robotic system including the manipulator and motors by applying the voltage control strategy. Stability analysis is presented and the effectiveness of the proposed control approach is demonstrated by simulations using a three link flexible-joint robot driven by permanent magnet DC motors. Simulation results show that the interval type-2 fuzzy PD controller can handle external disturbance better than the type-1 fuzzy PD controller. In addition, it spends less control effort than the type- 1 in order to deal with disturbance.
\end{abstract}

\section{Keywords}

Type-2 Fuzzy Control, Flexible-Joint Robots, Voltage Control Strategy, Uncertainty Estimation

\section{Introduction}

Fuzzy control as a model-free approach is simply designed to control complicated systems. In recent years, there has been an increasing attention to type 2 fuzzy logic system (FLS) in order to overcome the uncertainties. Type-1 FLS has difficulties in modeling and minimizing the effect of uncertainties [1]-[6]. This is because type- 1 fuzzy set is certain in the sense that the membership grade for a particular input is a crisp value [7]. Type-2 fuzzy sets are characterized by membership functions (MF) that are themselves fuzzy [1]-[6]. The type-2 FLS (T2FLS) 
can be used when circumstances are too uncertain to determine exact membership grades such as when training data is corrupted by noise. The most frequently used T2FLS is interval T2FLS (IT2FLS) for their reduced computational cost [4]. Though the T1FLS is the most widely used application of fuzzy set theory, the T2FLS have been used in a few control applications such as nonlinear control and mobile robot navigation [1], decision making [6], sliding mode control design [2] and chaotic control [8]. The direct and indirect adaptive interval type-2 fuzzy control has been proposed for nonlinear systems [5] [7]. However, an analytical proof should be given to guarantee stability and provide a desired performance.

Control of a flexible-joint robot as a complex system can highlight the capabilities of the T2FLS. In order to improve industrial productivity, it is required to reduce the weight of the arms and/or to increase their speed of operation. However, as a bad effect, the flexibility in joints and links may occur. On the other hand, compared to the conventional heavy and bulky robots, flexible manipulators have the potential advantage of lower cost, larger work volume, higher operational speed, greater payload-to-manipulator-weight ratio, smaller actuators, lower energy consumption, better maneuverability and better transportability due to reduced inertia [9] [10]. As a result, several applications such as space manipulators [10] [11] necessitate using flexible joint robots.

The most important reason of joint flexibility is the essential use of power transmission systems which show the flexibility [12]. The control schemes proposed for the rigid manipulators are limited in their applicability to real robots [13]. Compared with rigid robots, number of degrees of freedom in the flexible-joint robots becomes twice as number of control actions while the matching property between nonlinearities and inputs is lost [10] [12]. As a result, to improve the performance and avoid unwanted oscillations for practical applications, joint flexibility must be taken into account in both modeling and control [10]. During the past two decades, trajectory tracking control study of robotic manipulators with joint flexibilities has attracted considerable attention including singular perturbation theory [14], feedback linearization [15], adaptive control [16], sliding mode control [17], fuzzy control [18] and neural control [19]. The principal limitation existing in the aforementioned control schemes is that these schemes assume that torques can be directly applied to the robot links. In the other words, the control is designed at dynamic level with torque as input namely, torque control strategy while the actuator dynamics is excluded. However, the actuator dynamics represents an important component of complete electromechanical systems, especially in the case of high-velocity movement and highly varying loads [20] [21]. Furthermore, the additional sensing requirements, actuator saturation, and long processing time should be considered to implement the torque control strategy [12] [22]. As an example of torque control strategy [23], one can consider the complexity of the robust control of flexible-join robots. In some papers such as [20] the actuator dynamics is considered. 
However, the control problem may become more complicated due to considering the actuator dynamics.

To solve these problems, voltage control strategy has been devoted to the electrically driven robot manipulators [24]. In this strategy, the electric motors of the robot are controlled while the robot manipulator behaves as a load on the motors. Thus, a nominal model of the motor is required to design the controller with an advantage that the used model is simpler than the robot model. Recently, robust control [22] and nonlinear adaptive control [12] of flexible-joint robots have been developed using the voltage control strategy.

A model of robot may face uncertainties such as unmodelled dynamics, parametric uncertainty and external disturbances. In [25], the set-point regulation control design for an electrically driven flexible-joint robot with model uncertainty was proposed. In [16], an adaptive controller was developed to solve the tracking problem for electrically driven flexible-joint robot with time-varying uncertainty.

The contribution of this paper is to present an interval type-2 fuzzy PD (IT2PD) control approach for electrically driven flexible-joint robots. The proposed voltage control law has a simpler structure in the form of decentralized control yet more efficient than the torque control that is multivariable coupled control. As a result, the proposed control approach is free of many effects caused by manipulator dynamics. This is an important advantage of the proposed control approach over the torque based control approaches. Compared to the previous controllers reported in the literature for the flexible-joint robots which use two control loops, it has a simpler structure using only one control loop. This is the main novelty of this paper. Stability analysis is presented and the effectiveness of the proposed control approach is demonstrated by simulations.

The rest of the paper is organized as follows: Section 2 presents modeling of the flexible-joint robots. Section 3 introduces Interval type- 2 fuzzy logic. Section 4 develops the proposed method. Section 5 presents the simulation results and finally, Section 6 concludes the paper.

\section{Electrically Driven Flexible-Joint Robot Dynamics}

In a simplified model of the flexible-joint robot [23], the manipulator links are assumed rigid and motors are elastically coupled to the links. The motor torqueses are assumed as inputs of the robotic system. In this paper, the simplified model is applied for an electrically driven robot with some modifications to obtain them motor voltages as the inputs. Consider an electrical robot with revolute joints driven by the geared permanent magnet dc motors. If the joint flexibility is modeled by a linear torsional spring, the dynamic equation of motion can be expressed as [12] [22]

$$
\begin{aligned}
& \mathbf{D}(\boldsymbol{\theta}) \ddot{\boldsymbol{\theta}}+\mathbf{C}(\boldsymbol{\theta}, \boldsymbol{\theta}) \dot{\boldsymbol{\theta}}+\boldsymbol{g}(\boldsymbol{\theta})=\mathbf{K}\left(\mathbf{r} \boldsymbol{\theta}_{m}-\boldsymbol{\theta}\right) \\
& \mathbf{J} \ddot{\boldsymbol{\theta}}_{m}+\mathbf{B} \dot{\theta}_{m}+\mathbf{r K}\left(\mathbf{r} \theta_{m}-\theta\right)=\tau
\end{aligned}
$$


where $\boldsymbol{\theta} \in R^{n}$ is a vector of joint angles, $\boldsymbol{\theta}_{m} \in R^{n}$ is a vector of rotor angles. Thus, this system possesses $2 n$ coordinates as $\left[\begin{array}{ll}\boldsymbol{\theta} & \theta_{m}\end{array}\right]$. The matrix $\mathbf{D}(\boldsymbol{\theta})$ is a $n \times n$ matrix of manipulator inertia, $\mathbf{C}(\boldsymbol{\theta}, \boldsymbol{\theta}) \dot{\boldsymbol{\theta}} \in R^{n}$ is the vector of centrifugal and Coriolis forces, $\boldsymbol{g}(\boldsymbol{\theta}) \in R^{n}$ is a vector of gravitational forces and $\tau \in R^{n}$ is a torque vector of motors. The diagonal matrices $\mathbf{J , B}$ and $\mathbf{r}$ represent coefficients of the motor inertia, motor damping and reduction gear, respectively. The diagonal matrix $\mathbf{K}$ represents the lumped flexibility provided by the joint and reduction gear. To simplify the model, both the joint stiffness and gear coefficients are assumed constant. The vector of gravitational forces $g(\theta)$ is assumed function of only the joint positions as used in the simplified model [24]. Note that the vector and matrix are represented in bold form for clarity.

System (1)-(2) is highly nonlinear, extensively computational, heavily coupled and multi-input/multi-output system with the $2 n$ coordinates. Complexity of the model has been a serious challenge in robot modeling and control in literature. It is expected to face a higher complexity if the proposed model includes the actuator dynamics. In order to obtain the motor voltages as inputs, consider electrical equation of the geared permanent magnet dc motors in the matrix form

$$
\mathbf{u}=\mathbf{R} \boldsymbol{I}_{a}+\mathbf{L} \dot{\boldsymbol{I}}_{a}+\mathbf{K}_{b} \dot{\boldsymbol{\theta}}_{m}
$$

where $\boldsymbol{u} \in R^{n}$ is a vector of motor voltages, $\boldsymbol{I}_{a} \in R^{n}$ is a vector of motor currents and $\dot{\theta}_{m}$ is a vector of rotor velocities. The diagonal matrices $\mathbf{R}, \mathbf{L}$ and $\mathbf{K}_{b}$ represent the coefficients of armature resistance, armature inductance and back-emf constant, respectively. The motor torques $\tau$ as input for dynamic Equation (2) is produced by the motor currents as

$$
\mathbf{K}_{m} \boldsymbol{I}_{a}=\boldsymbol{\tau}
$$

where $\mathbf{K}_{m}$ is a diagonal matrix of the torque constants. Equations (1)-(4) form the robotic system such that the voltage vector $\boldsymbol{u}$ is the input vector and the joint angle vector $\theta$ is the output vector.

The dynamics of the electrical robot (1)-(4) in the state space is formed as

$$
\dot{\mathrm{x}}=\boldsymbol{f}(\mathrm{x})+\boldsymbol{b u}
$$

where

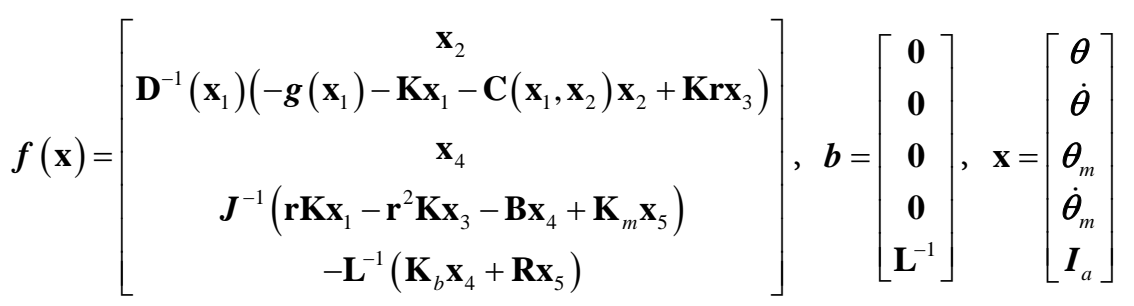

\section{Interval Type-2 Fuzzy Logic System}

A fuzzy logic system that uses at least one type-2 fuzzy set is called a type-2 fuzzy logic system. It is very useful in circumstances where determination of an exact membership grade for a fuzzy set is difficult [4]. As illustrated in Figure 1, a 
type-2 fuzzy membership function (MF) can be obtained by starting with a type-1 MF and blurring it. The extra mathematical dimension provided by the blurred area, referred to as the footprint of uncertainty (FOU), and represents the uncertainties in the shape and position of the type- 1 fuzzy set. The FOU is bounded by upper and lower MF, and points within the "blurred area" have membership grades given by type-1 MF. The most frequently used type- 2 fuzzy sets are interval fuzzy sets where each point in the FOU has unity secondary membership grade [3].

An interval type-2 fuzzy set $\tilde{\mathrm{A}}$ in $\mathrm{X}$ is defined as [4]:

$$
\tilde{\mathrm{A}}=\int_{x \in \mathrm{X}}\left[\int_{u \in J_{x}} \frac{1}{u}\right] / x, J_{x} \subseteq[0,1]
$$

where $x$ is the primary variable with domain $\mathrm{X}$; $u$ is the secondary variable, which has domain $J_{x} ; J_{x}$ is called the primary membership of $x$. Uncertainty about $\tilde{A}$ is conveyed by the union of all of the primary memberships called the footprint of uncertainty (FOU) of $\tilde{\mathrm{A}}$; i.e.

$$
\operatorname{FOU}(\tilde{\mathrm{A}})=J_{x}, x \in \mathrm{X}
$$

The structure of a typical type- 2 fuzzy logic system is shown in Figure 2 It is similar to its type-1 counterpart, the major difference being that at least one of the fuzzy sets is type-2 and a type-reducer is needed to convert the type- 2 fuzzy output sets into type- 1 sets so that they can be processed by the defuzzifier to give a crisp output. General type-2 FLSs are computationally intensive because type-reduction is very intensive [4]. Therefore, we will use in this work the interval type-2 fuzzy logic systems for their simplicity and efficiency.

In the following subsections the operations in an interval singleton type-2 FLS are described in details.

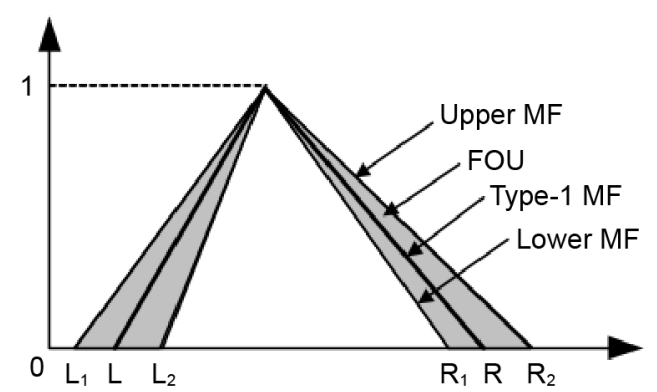

Figure 1. Type-2 fuzzy logic membership function.

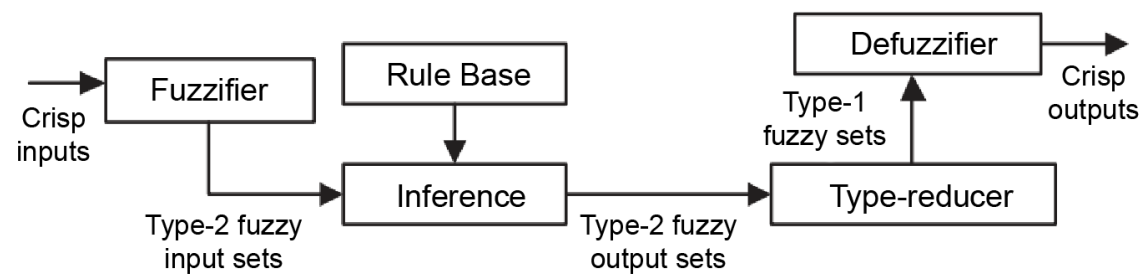

Figure 2. Scheme of a type-2 fuzzy logic system. 


\subsection{Fuzzification}

In practice the computations in an IT2FLS can be consisting of $M$ rules assuming the following form:

$$
R^{i}: \text { if } x_{1} \text { is } \tilde{\mathrm{X}}_{1}^{i} \text { and } \cdots x_{p} \text { is } \tilde{\mathrm{X}}_{p}^{i} \text {, then } y \text { is } \mathrm{Y}^{i}, i=1,2, \cdots, M
$$

where $\mathrm{x}=\left(x_{1}, \cdots, x_{p}\right)$ is the input vector, $y$ are linguistic variables, $\tilde{\mathrm{X}}_{j}^{i}(j=1,2, \cdots, p)$ is an interval type-2 fuzzy set and $\mathrm{Y}^{i}=\left[y_{l}^{i}, y_{r}^{i}\right]$, which can be understood as the simplest Takagi-Sugeno-Kang (TSK) model. The fuzzifier maps a crisp point $\mathrm{x}=\left(x_{1}, \cdots, x_{p}\right)$ into a type-2 fuzzy set $\tilde{\mathrm{A}}$ [26].

\subsection{Inference}

The inference engine matches the fuzzy singletons with the fuzzy rules in the rule base. To compute unions and intersections of type- 2 sets, compositions of type-2 relations are needed [9]. The first step in the extended sup-star operation is to obtain the firing set $\prod_{j=1}^{p} \mu_{\tilde{\mathrm{X}}_{j}^{i}}=\mathrm{F}^{i}(\mathrm{x})$ by performing the input and antecedent operations. As only interval type- 2 sets are used and the meet operation is implemented by the product t-norm, the firing set is the following type- 1 interval set [26]:

$$
\mathrm{F}^{i}(\mathrm{x})=\left[\underline{f}^{i}(\mathrm{x}), \bar{f}^{i}(\mathrm{x})\right]=\left[\underline{f}^{i}, \bar{f}^{i}\right]
$$

where $\underline{f}^{i}(\mathrm{x})=\underline{\mu}_{\tilde{\mathrm{X}}_{1}^{i}}\left(x_{1}\right) \times \cdots \times \underline{\mu}_{\tilde{\mathrm{X}}_{p}^{i}}\left(x_{p}\right)$ and $\bar{f}^{i}(\mathrm{x})=\bar{\mu}_{\tilde{\mathrm{X}}_{1}^{i}}\left(x_{1}\right) \times \cdots \times \bar{\mu}_{\tilde{\mathrm{X}}_{p}^{i}}\left(x_{p}\right)$ the terms $\underline{\mu}_{\tilde{\mathrm{X}}_{j}^{i}}$ and $\bar{\mu}_{\tilde{\mathrm{X}}_{j}^{i}}$ are the lower and upper membership grades of $\mu_{\tilde{\mathrm{x}}_{j}^{i}}$, respectively.

\subsection{Type-Reduction}

The type- 2 fuzzy inference engine produces an aggregated output type- 2 fuzzy set. The type reduction block operates on this set to generate a centroid type-1 fuzzy set known as the "type-reduced set" of the aggregate type-2 fuzzy set. Several type-reduction methods have been suggested in the literature, such as the center-of-sums, the height, the modified height and the center-of-sets, for example [4] [5] [26]. In this article, we consider the center-of-sets type reduction technique due to its computational efficiency. That may be expressed as [3]

$$
\mathrm{Y}_{\text {cos }}(\mathrm{x})=\left[y_{l}, y_{r}\right]=\bigcup_{\substack{f^{i} \in \mathrm{i}^{i}(\mathrm{x}) \\ y^{i} \in \mathrm{Y}^{i}}} \frac{\sum_{i=1}^{N} f^{i} y^{i}}{\sum_{i=1}^{M} f^{i}}
$$

where $\mathrm{Y}_{c o s}$ is the interval set determined by two end points $y_{l}$ and $y_{r}$, and firing strengths $f^{i}=\left[\underline{f}^{i}, \bar{f}^{i}\right] \in \mathrm{F}^{i}(\mathrm{x}), y_{l}$ and $y_{r}$ can be expressed as [27]:

$$
\begin{aligned}
& y_{l}=\frac{\sum_{i=1}^{L} \bar{f}^{i} y_{l}^{i}+\sum_{i=L+1}^{N} \underline{f}^{i} y_{l}^{i}}{\sum_{i=1}^{L} \bar{f}^{i}+\sum_{i=L+1}^{N} \underline{f}^{i}} \\
& y_{r}=\frac{\sum_{i=1}^{R} \frac{f^{i}}{y_{r}^{i}+\sum_{i=R+1}^{N} \bar{f}^{i} y_{r}^{i}}}{\sum_{i=1}^{R} \underline{f}^{i}+\sum_{r=R+1}^{N} \bar{f}^{i}}
\end{aligned}
$$


Two end points $y_{l}$ and $y_{r}$ can be computed efficiently using the Karnik-Mendel (KM) algorithms [28]. For example The EKM algorithm for computing $y_{r}$ is given as:

1) Sort $\underline{x}_{j}=(i=1, \cdots, N)$ in increasing order

2) Initialize $f^{i}$ by setting $f^{i}=\frac{f^{i}+\bar{f}^{i}}{2}(i=1,2, \cdots, N)$ and then compute $y=\frac{\sum_{i=1}^{N} x_{j} f^{i}}{\sum_{i=1}^{N} f^{i}}$

3) Find switch point $k(1 \leq k \leq N-1)$ such that $\underline{x}_{k} \leq y \leq \bar{x}_{k}+1$

4) Set $f^{i}=\bar{f}^{i}(i \leq k)$ and $f^{i}=\underline{f}^{i}(i>k)$ then compute $y^{\prime}=\frac{\sum_{i=1}^{N} \underline{x}_{i} f^{i}}{\sum_{i=1}^{N} f^{i}}$

5) Check if $y^{\prime}=y$. If yes, stop, set $y_{l}=y$, and call $k, L$. If no go to step 6

6) set $y=y^{\prime}$ and go to step 3

\subsection{Defuzzification}

Since the type-reduced set is an interval type-1 set, the defuzzified output is [27]:

$$
y(\mathrm{x})=0.5\left(y_{l}+y_{r}\right)
$$

\section{Proposed Control Law}

To control such a complicated system a novel simple controller is proposed using voltage control strategy. Electrical equation of a permanent magnet dc motor is written as

$$
u=R I_{a}+L \dot{I}_{a}+k_{b} \dot{\theta}_{m}+\varphi
$$

where $R, L$ and $k_{b}$ denote the armature resistance, inductance, and back emf constant, respectively. $u$ is the motor voltage, $I_{a}$ motor current, and $\theta_{m}$ the rotor position. $\varphi$ represents the external disturbance.

The motor angle $\theta_{m}$ as an output can be controlled via the voltage $u$ as an input. It is very interesting to note that (7) is a single-input/single-output (SISO) system while the robot manipulator is a multivariable multi-input system. The motor current $I_{a}$ contains effects of coupling between the motor and the manipulator.

From (2), we have

$$
\mathrm{r} \theta_{m}=\mathrm{K}^{-1}\left(\tau_{l}\right)+\theta
$$

In addition, Equation (3) can be rewritten as

$$
\tau_{l}=\mathrm{r}^{-1}\left(\tau+\varphi_{1}\right)
$$

where $\varphi_{1}$ include unmodeled dynamics. Substituting (16) into (15) and using (5) yields

$$
\mathrm{r} \theta_{m}=\mathrm{K}^{-1}\left(\mathrm{r}^{-1}\left(\mathrm{~K}_{m} I_{a}+\varphi_{1}\right)\right)+\theta
$$

Taking the time derivative of the above equation yields 


$$
\dot{\theta}_{m}=\mathrm{K}^{-1} \mathrm{r}^{-2} \mathrm{~K}_{m} \dot{I}_{a}+\mathrm{r}^{-1} \dot{\theta}+\mathrm{K}^{-1} \mathrm{r}^{-2} \dot{\varphi}_{1}
$$

Substituting (18) into (14) gives

$$
u=L_{m} \dot{I}_{a}+k_{b} \mathrm{r}^{-1} \dot{\theta}+\varphi_{2}
$$

where $L_{m}=L+k_{b} \mathrm{~K}^{-1} \mathrm{r}^{-2} \mathrm{~K}_{m}$ and $\varphi_{2}=k_{b} \mathrm{~K}^{-1} \mathrm{r}^{-2} \dot{\varphi}_{1}+R I_{a}+\varphi$

The current of the motor can be directly controlled using a PI controller as follows:

$$
I_{a}=k_{p} e+k_{i} \int e \mathrm{~d} t
$$

where $k_{d}$ and $k_{i}$ are positive constant gains. $e$ is tracking error expressed by $e=\theta_{d}-\theta$. In the meantime, $\theta$ is the actual joint angle and $\theta_{d}$ is the desired joint.

Substituting (20) into (19) yields

$$
u=L_{m}\left(k_{p} \dot{e}+k_{i} e\right)+k_{b} \mathrm{r}^{-1}(\dot{\theta})+\varphi_{2}
$$

Using (21) a control law is proposed as

$$
u=L_{m}\left(k_{p} \dot{e}+k_{i} e\right)+k_{b} \mathrm{r}^{-1}\left(\dot{\theta}_{d}+\beta\left(\theta_{d}-\theta\right)\right)+\hat{\varphi}_{2}
$$

where $\beta$ is a positive constant and $\hat{\varphi}_{2}$ is the estimation of $\varphi_{2}$. After some manipulation, one can obtain

$$
u=k_{b} r^{-1} \dot{\theta}_{d}+\left(k_{p}^{\prime}\right) e+\left(k_{d}^{\prime}\right) \dot{e}+\hat{\varphi}_{2}
$$

where $k_{p}^{\prime}=L_{m} k_{i}+k_{b} r^{-1} \beta$ and $k_{d}^{\prime}=L_{m} k_{p}$.

Equation (23) includes three terms. The first term is $k_{b} r^{-1} \dot{\theta}_{d}$ and the second term can be considered as a PD controller. The third term is the estimation of uncertainty. As a result, we can conclude from (23) that a flexible joint robot can be controlled directly using a simple PD controller plus uncertainty estimation with an extra term expressed by $k_{b} r^{-1} \dot{\theta}_{d}$. It should be stated that the IT2FLC can be used instead of the PD control plus uncertainty estimation. This is why the IT2FLC can handle the uncertainty. Compared to the previous controllers reported for the flexible-joint robots which use two control loops, it has a simpler structure and more efficiency using only one control loop.

Suppose that $y$ is the output of an IT2PD in the normalized form with the inputs of $x_{1}$ and $x_{2}$. If three fuzzy sets are given to each fuzzy input, the whole control space will be covered by nine fuzzy rules. The linguistic fuzzy rules are proposed as

$$
R^{i} \text { : if } x_{1} \text { is } \tilde{\mathrm{X}}_{1}^{i} \text { and } x_{2} \text { is } \tilde{\mathrm{X}}_{2}^{i} \text { then } y^{i}=a_{i 1} x_{1}+a_{i 2} x_{2}+a_{i 0}, i=1,2, \cdots, 9
$$

where $R^{i}$ denotes the $i$ th fuzzy rule for $i=1, \cdots, 9$. In the $i$ th rule, $\tilde{\mathrm{X}}_{1}^{i}$ and $\tilde{\mathrm{X}}_{2}^{i}$ are type-2 fuzzy membership functions belonging to the fuzzy variables $x_{1}$ and $x_{2}$, respectively. $a_{i 1}, a_{i 2}$ and $a_{i 0}$ are the gain in consequent part and $y^{i}$ is the crisp output. The proposed interval type-2 fuzzy controller is for the case when antecedents are interval type-2 fuzzy sets (A2) and consequents are crisp numbers (C0). Three Gaussian membership functions with uncertain mean, $\mu_{\tilde{\mathrm{X}}_{1}^{i}}\left(x_{1}\right)$, named as Positive $(\mathrm{P})$, Zero $(\mathrm{Z})$, and Negative $(\mathrm{N})$ are defined for input 
$x_{1}$ in the operating range of manipulator as shown in Figure 3. Three Gaussian membership functions with uncertain mean, $\mu_{\tilde{\mathrm{X}}_{2}^{i}}\left(x_{2}\right)$, named as $\mathrm{P}, \mathrm{Z}$, and $\mathrm{N}$ in the same shape as Figure 3, are used for input $x_{2}$. As shown in Figure 3 all universes of discourses are normalized and arranged in [-1 1] with scaling factors external to the FLC used to give appropriate values to the variables. The role of input scaling factors becomes more important for using the Gaussian MFs for inputs. The input scaling factors are employed to take the input into the operating range covered by MFs otherwise the controller will not respond to the input. Input variables and output variable have scaling factors $\mathrm{k}_{p_{1}}, \mathrm{k}_{d_{1}}$ and $\mathrm{k}_{\mathrm{o}_{1}}$ for joint $1, \mathrm{k}_{p_{2}}, \mathrm{k}_{d_{2}}$ and $\mathrm{k}_{\mathrm{o}_{2}}$ for joint 2, $\mathrm{k}_{p_{3}}, \mathrm{k}_{d_{3}}$ and $\mathrm{k}_{\mathrm{o}_{3}}$ for joint 3 ,respetively. The IT2PD controllers should be designed in such a way that the stability of control system is guaranteed.

\section{Stability Analysis of the Control System}

In other words, $y_{l}$ in (11) can be rewritten as

$$
y_{l}=\sum_{i=1}^{L} \bar{q}_{l}^{i}\left(a_{i 1} x_{1}+a_{i 2} x_{2}+a_{i 0}\right)+\sum_{i=L+1}^{M} \underline{q}_{l}^{i}\left(a_{i 1} x_{1}+a_{i 2} x_{2}+a_{i 0}\right)
$$

where $\bar{q}_{l}^{i}=\bar{f}^{i} / D_{l}$ and $q_{l}^{i}=f^{i} / D_{l}$. In the meantime, we have $D_{l}=\sum_{i=1}^{L} \bar{f}^{i}+\sum_{i=L+1}^{M} \underline{f}^{i}$.

In the similar manner, $y_{r}$ in (12) can be rewritten as

$$
y_{r}=\sum_{i=1}^{R} \underline{q_{r}^{i}}\left(a_{i 1} x_{1}+a_{i 2} x_{2}+a_{i 0}\right)+\sum_{i=R+1}^{M} \bar{q}_{r}^{i}\left(a_{i 1} x_{1}+a_{i 2} x_{2}+a_{i 0}\right)
$$

where $\bar{q}_{r}^{i}=\bar{f}^{i} / D_{r}$ and $q_{r}^{i}=f^{i} / D_{r}$. In the meantime, we have $D_{r}=\sum_{i=1}^{R} \underline{f}^{i}+\sum_{i=R+1}^{M} \bar{f}^{i}$.

From (13) after some manipulation, one can obtain

$$
y(\mathrm{x})=\mathrm{C}_{1}(\mathrm{x}) x_{1}+\mathrm{C}_{2}(\mathrm{x}) x_{2}+\mathrm{C}_{0}(\mathrm{x})
$$

where

$$
\begin{aligned}
& \mathrm{C}_{1}(\mathrm{x})=0.5\left(\sum_{i=1}^{L} \bar{q}_{l}^{i} a_{i 1}+\sum_{i=L+1}^{M} \bar{q}_{l}^{i} a_{i 1} \sum_{i=1}^{R} \bar{q}_{r}^{i} a_{i 1}+\sum_{i=R+1}^{M} \bar{q}_{r}^{i} a_{i 1}\right) \\
& \mathrm{C}_{2}(\mathrm{x})=0.5\left(\sum_{i=1}^{L} \bar{q}_{l}^{i} a_{i 2}+\sum_{i=L+1}^{M} \bar{q}_{l}^{i} a_{i 2}+\sum_{i=1}^{R} \bar{q}_{r}^{i} a_{i 2}+\sum_{i=R+1}^{M} \bar{q}_{r}^{i} a_{i 2}\right) \\
& \mathrm{C}_{0}(\mathrm{x})=0.5\left(\sum_{i=1}^{L} \bar{q}_{l}^{i} a_{i 0}+\sum_{i=L+1}^{M} \bar{q}_{l}^{i} a_{i 0}+\sum_{i=1}^{R} \bar{q}_{r}^{i} a_{i 0}+\sum_{i=R+1}^{M} \bar{q}_{r}^{i} a_{i 0}\right)
\end{aligned}
$$

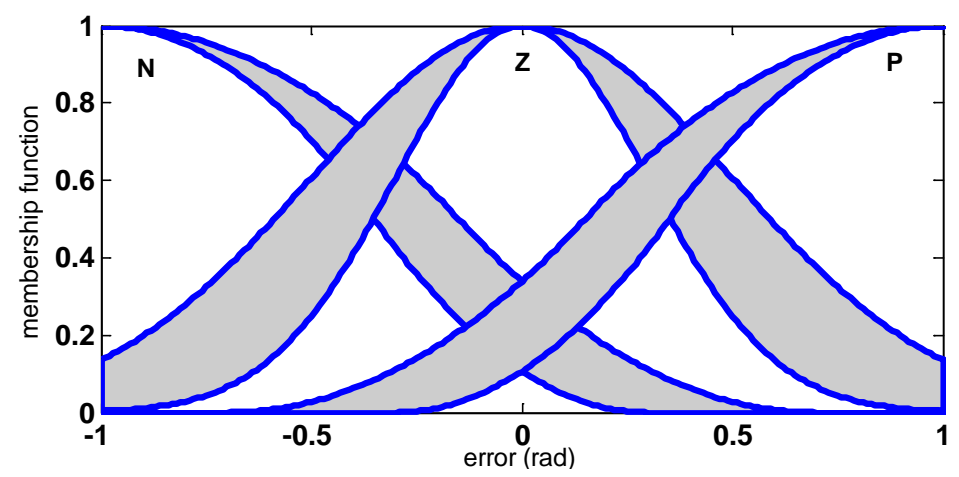

Figure 3. Membership function of the input e. 
The obtained analytical structure of the fuzzy controller improves our study to develop the analysis and design. Using the scaling factors the input vector is formed as

$$
\mathrm{x}=\left[\mathrm{k}_{p_{i}} z_{1} \mathrm{k}_{d_{i}} z_{2}\right]^{\mathrm{T}}
$$

where for the ith joint $z_{1}$ and $z_{2}$ are defined as

$$
\begin{aligned}
& \mathrm{x}=\left[\mathrm{k}_{p_{i}} \mathrm{z}_{1} \mathrm{k}_{d_{i}} \mathrm{z}_{2}\right]^{\mathrm{T}} \\
& \mathrm{z}_{1}=\theta_{d i}-\theta_{i} \\
& \mathrm{z}_{2}=\dot{\theta}_{d i}-\dot{\theta}_{i}
\end{aligned}
$$

where $\theta_{d i}$ and $\theta_{i}$ are the desired and actual joint position, respectively. From (32) and (33) we have $\dot{z}_{1}=z_{2}$.

Using $x_{1}=\mathrm{k}_{p_{i}} \mathrm{z}_{1}$ and $x_{2}=\mathrm{k}_{d_{i}} \mathrm{z}_{2}$, one can obtain

$$
\dot{x}_{1}=\alpha x_{2}
$$

where $\alpha=\mathrm{k}_{p_{i}} / \mathrm{k}_{d_{i}}>0$.

Fuzzy controller by the use of scaling factors is formed as

$$
\mathrm{u}(\mathrm{x})=\mathrm{k}_{0_{i}}\left(\mathrm{C}_{1}(\mathrm{x}) x_{1}+\mathrm{C}_{2}(\mathrm{x}) x_{2}+\mathrm{C}_{0}(\mathrm{x})\right)+k_{b} r^{-1} \dot{\theta}_{d}
$$

This general structure shows a nonlinear variable gain controller that finds many applications in control. The nonlinear gain $\mathrm{C}_{\mathrm{i}}(\mathrm{x})$ covers the nonlinearity of controller by parameters in hand. The control purposes are simply described by linguistic rules in fuzzy controller transformed to a nonlinear function as stated by (35).

Substituting (35) into (3) forms the closed loop system as follows

$$
\left(\mathrm{C}_{1}(\mathrm{x}) x_{1}+\mathrm{C}_{2}(\mathrm{x}) x_{2}+\mathrm{C}_{0}(\mathrm{x})\right) \mathrm{k}_{0_{i}}+k_{b} r^{-1} \dot{\theta}_{d}=R I_{a}+L \dot{I}_{a}+k_{b} \dot{\theta}_{m}
$$

Assume that the motor voltage $u$ expressed by (3) is limited such that

$$
\left|R I_{a}+L \dot{I}_{a}+k_{b} \dot{\theta}_{m}\right| \leq u_{\max }
$$

where $u_{\max }>0$ is a maximum permitted voltage for the motor. This assumption is a technical regard to protect motor against over voltages. The complexity of design and analysis has been changed to simplicity for using the model of motor in place of model of manipulator. Here, we should know only the upper limits for the motor voltages as inputs of robotic system. Because electrical motors drive the electrical manipulator, the motor voltages are the system inputs. The desired trajectory should be planned with regarding the maximum permitted voltages for motors somehow each motor is so strong such that can track the desired trajectory under the permitted voltage. Moreover, the desired trajectory should be smooth such that its derivatives up to the required order are available and limited. To find a control law for the convergence of error, a positive definite function is proposed as

$$
\mathrm{V}=\int_{0}^{x_{1}} \mathrm{C}_{2}(\mathrm{x}) x_{1} \mathrm{~d} x_{1}
$$


where $\mathrm{V}$ is a positive definite function of $x_{1}$ if $\mathrm{C}_{2}(\mathrm{x})$ is positive. To satisfy $0 \leq \mathrm{C}_{2}(\mathrm{x})$ it is sufficient that $0 \leq a_{i 2}$.

Proof: Assume that $0<\mathrm{C}_{2}<\mathrm{C}_{2}(\mathrm{x})$ where $\mathrm{C}_{2}$ is a positive constant. Thus,

$$
\mathrm{C}_{2} \int_{0}^{x_{1}} x_{1} \mathrm{~d} x_{1}<\int_{0}^{x_{1}} \mathrm{C}_{2}(\mathrm{x}) x_{1} \mathrm{~d} x_{1}
$$

we have $\int_{0}^{x_{1}} \mathrm{C}_{2} x_{1} \mathrm{~d} x_{1}=0.5 \mathrm{C}_{2} x_{1}^{2}$. Hence, $0.5 \mathrm{C}_{2} x_{1}^{2}<\int_{0}^{x_{1}} \mathrm{C}_{2}(\mathrm{x}) x_{1} \mathrm{~d} x_{1}$. Thus (39) implies that $\mathrm{V}>0$ for $x_{1} \neq 0$. Since $\int_{0}^{0} \mathrm{C}_{2}(\mathrm{x}) x_{1} \mathrm{~d} x_{1}=0$ and $\mathrm{C}_{2}(\mathrm{x}) x_{1}$ is limited, $\mathrm{V}=0$ if $x_{1}=0$. Thus, $\mathrm{V}$ is a positive definite function of $x_{1}$.

The time derivative of $\mathrm{V}$ is calculated as

$$
\dot{\mathrm{V}}=\mathrm{C}_{2}(\mathrm{x}) x_{1} \dot{x}_{1}=\alpha \mathrm{C}_{2}(\mathrm{x}) x_{1} x_{2}
$$

From (36) we can write

$$
\mathrm{C}_{2}(\mathrm{x}) x_{2}=-\mathrm{C}_{1}(\mathrm{x}) x_{1}-\mathrm{C}_{0}(\mathrm{x})+\left(R I_{a}+L \dot{I}_{a}+k_{b} \dot{\theta}_{m}\right) / \mathrm{k}_{0_{i}}-k_{b} r^{-1} \dot{\theta}_{d} / \mathrm{k}_{0_{i}}
$$

Substituting (41) into (40) yields

$$
\dot{\mathrm{V}}=-\alpha \mathrm{C}_{1}(\mathrm{x}) x_{1}^{2}-\alpha \mathrm{C}_{0}(\mathrm{x}) x_{1}+\alpha x_{1}\left(R I_{a}+L \dot{I}_{a}+k_{b} \dot{\theta}_{m}\right) / \mathrm{k}_{0_{i}}-\alpha x_{1} k_{b} r^{-1} \dot{\theta}_{d} / \mathrm{k}_{0_{i}}
$$

Since $-\alpha \mathrm{C}_{1}(\mathrm{x}) x_{1}^{2} \leq 0$ for $0 \leq \mathrm{C}_{1}(\mathrm{x})$, to satisfy $\dot{\mathrm{V}} \leq 0$ for stability, it is required that

$$
x_{1}\left(R I_{a}+L \dot{I}_{a}+k_{b} \dot{\theta}_{m}\right)-x_{1} k_{b} r^{-1} \dot{\theta}_{d} \leq \mathrm{k}_{0_{i}} \mathrm{C}_{0}(\mathrm{x}) x_{1}
$$

Using the Cauchy-Schwartz inequality, one can obtain

$$
\begin{aligned}
& x_{1}\left(R I_{a}+L \dot{I}_{a}+k_{b} \dot{\theta}_{m}\right)-x_{1} k_{b} r^{-1} \dot{\theta}_{d} \\
& \leq\left|x_{1}\right|\left|R I_{a}+L \dot{I}_{a}+k_{b} \dot{\theta}_{m}\right|+k_{b} r^{-1}\left|x_{1}\right|\left|\dot{\theta}_{d}\right| \leq\left|x_{1}\right| u_{\max }
\end{aligned}
$$

Suppose that $\left|\dot{\theta}_{d}\right| \leq \gamma$ where $\gamma$ is a positive constant. To satisfy (44), it is sufficient that

$$
\left|x_{1}\right|\left(u_{\max }+k_{b} r^{-1} \gamma\right) \leq \mathrm{k}_{0_{i}} \mathrm{C}_{0}(\mathrm{x}) x_{1}
$$

Since $\mathrm{k}_{0}>0$, to guarantee stability $0<x_{1} \mathrm{C}_{0}(\mathrm{x})$. This means that $\mathrm{C}_{0}(\mathrm{x})$ must be designed with the same sign as $x_{1}$. This condition is simply satisfied if $a_{i 0}$ is selected with the same sign as $x_{1}$.

From (45) and $x_{1} \mathrm{C}_{0}(\mathrm{x})>0$, we obtain

$$
\left(u_{\max }+k_{b} r^{-1} \gamma\right) /\left|\mathrm{C}_{0}(\mathrm{x})\right| \leq \mathrm{k}_{0_{i}}
$$

From (30), one can obtain

$$
\mathrm{c}_{0, \min } \leq \mathrm{C}_{0}(\mathrm{x}) \leq \mathrm{c}_{0, \max }
$$

where $c_{0, \min }$ and $c_{0, \max }$ are to constant. To select a constant value, we should select a value for $\mathrm{k}_{0}$ that satisfies (46) in all cases. The maximum permitted value for $k_{0}$ is then selected as

$$
\left(u_{\max }+k_{b} r^{-1} \gamma\right) / \mathrm{c}_{0, \max }=\mathrm{k}_{0_{i}}
$$

Therefore, stability is guaranteed under assumptions $\mathrm{C}_{1}(\mathrm{x})>0, \mathrm{C}_{2}(\mathrm{x})>0$, $x_{1} \mathrm{C}_{0}(\mathrm{x})>0$ and $\left(u_{\max }+k_{b} r^{-1} \gamma\right) / \mathrm{c}_{0, \max }=\mathrm{k}_{0_{i}}$. 
In the ith rule, $a_{i 0}$ is selected with the same sign as $x_{1}$ to satisfy $x_{1} \mathrm{C}_{0}(\mathrm{x})>0$. We can select $a_{i 1} \geq 0$ and $a_{i 2} \geq 0$ in all subsections but $\exists i$ that $a_{i 1}>0$ and $a_{i 2}>0$ to satisfy $\mathrm{C}_{2}(\mathrm{x})>0, x_{1} \mathrm{C}_{0}(\mathrm{x})>0$, respectively.

Fuzzy rules in the 9 subsections for $i=1, \cdots, 9$ are designed where the following cases occur:

Case 1 Assume that $x_{1} x_{2}<0$ resulting in asymptotic stability by $\dot{\mathrm{V}}<0$ in (40). Thus, a small control effort is given to $u$.

Case 2 Assume that $x_{1}=0$ or $x_{2}=0$ resulting in Lyapunov stability by $\dot{\mathrm{V}}=0$ in (40). Thus, a medium control effort is given to $u$.

Case 3 Assume that $x_{1}$ and $x_{2}$ both are positive or negative resulting in instability by $\dot{\mathrm{V}}>0$ in (40). Thus, a very high effort is given to $u$.

The fuzzy rules for the first and second controllers are then given as:

Rule 1: if $x_{1}$ is $\mathrm{P}$ and $x_{2}$ is $\mathrm{P}$ then $y=1$

Rule 2 : if $x_{1}$ is $\mathrm{P}$ and $x_{2}$ is $\mathrm{Z}$ then $y=0.75$

Rule 3: if $x_{1}$ is $\mathrm{P}$ and $x_{2}$ is $\mathrm{N}$ then $y=0.25$

Rule 4 : if $x_{1}$ is $\mathrm{Z}$ and $x_{2}$ is $\mathrm{P}$ then $y=0.5$

Rule 5: if $x_{1}$ is Z and $x_{2}$ is $Z$ then $y=150 x_{1}+10 x_{2}$

Rule 6 : if $x_{1}$ is $\mathrm{Z}$ and $x_{2}$ is $\mathrm{N}$ then $y=-0.5$

Rule 7 : if $x_{1}$ is $\mathrm{N}$ and $x_{2}$ is $\mathrm{P}$ then $y=-0.25$

Rule 8: if $x_{1}$ is $\mathrm{N}$ and $x_{2}$ is $\mathrm{Z}$ then $y=-0.75$

Rule 9: if $x_{1}$ is $\mathrm{N}$ and $x_{2}$ is $\mathrm{N}$ then $y=-1$

Therefore, using the above analysis the $x_{1}, x_{2}$ are bounded. Then one can imply the boundedness of $u$ because of boundedness $x_{1}$ and $x_{2}$.

Proof: From (28), $C_{1}(x), C_{2}(x)$ and $\left|C_{0}(x)\right|$ are bounded as

$$
\begin{aligned}
& \left|C_{1}(x)\right| \leq 0.5 \alpha_{1} \\
& \left|C_{2}(x)\right| \leq 0.5 \alpha_{2} \\
& \left|C_{0}(x)\right| \leq 0.5 \alpha_{0}
\end{aligned}
$$

where

$$
\begin{aligned}
& \left|\sum_{i=1}^{L} \bar{q}_{l}^{i} a_{i 1}+\sum_{i=L+1}^{M} \bar{q}_{l}^{i} a_{i 1}+\sum_{i=1}^{R} \bar{q}_{r}^{i} a_{i 1}+\sum_{i=R+1}^{M} \bar{q}_{r}^{i} a_{i 1}\right| \leq \alpha_{1} \\
& \left|\sum_{i=1}^{L} \bar{q}_{l}^{i} a_{i 2}+\sum_{i=L+1}^{M} \bar{q}_{l}^{i} a_{i 2}+\sum_{i=1}^{R} \bar{q}_{r}^{i} a_{i 2}+\sum_{i=R+1}^{M} \bar{q}_{r}^{i} a_{i 2}\right| \leq \alpha_{2} \\
& \left|\sum_{i=1}^{L} \bar{q}_{l}^{i} a_{i 0}+\sum_{i=L+1}^{M} \bar{q}_{l}^{i} a_{i 0}+\sum_{i=1}^{R} \bar{q}_{r}^{i} a_{i 0}+\sum_{i=R+1}^{M} \bar{q}_{r}^{i} a_{i 0}\right| \leq \alpha_{0}
\end{aligned}
$$

where $\alpha_{1}, \alpha_{2}$ and $\alpha_{0}$ are constant.

Considering (9) we have

$$
\begin{aligned}
& \underline{f}^{i}(x) \leq 1 \\
& \bar{f}^{i}(x) \leq 1
\end{aligned}
$$


Thus, one can imply that $\bar{q}_{l}^{i}, \underline{q}_{l}^{i}$ and $\bar{q}_{r}^{i}, \underline{q}_{r}^{i}$ are bounded. The coefficient $a_{i 1}$ is a constant parameter. As a result, the inequality (52) is verified.

Similarly, the inequality (53) and (54) are proven. Therefore, $u$ is bounded using (35) as

$$
|u| \leq \mathrm{k}_{0_{i}}\left(\alpha_{1}\left|x_{1}\right|+\alpha_{2}\left|x_{2}\right|+\alpha_{0}\right)+k_{b} r^{-1} \gamma
$$

According to the proof given by [22], since the input $u$ is bounded variable $I_{a}$ is bounded.

Since the desired joint angle $\theta_{d}$ and its time derivative $\dot{\theta}_{d}$ are bounded. The bound variables $x_{1}$ and $x_{2}$ imply that $\theta=\theta_{d}-x_{1}$ and $\dot{\theta}=\dot{\theta}_{d}-x_{2}$ are bounded.

Since $I_{a}$ is bounded, (4) implies that $\tau$ is bounded. From (2) we have

$$
J \ddot{\theta}_{m}+B \dot{\theta}_{m}+r^{2} K \theta_{m}=\tau+r K \theta
$$

System (58) is a second order linear system with positive gains $B, r^{2} K$, and a limited input $\tau+r K \theta$.This system is stable based on the Routh-Hurwitz criterion and implies that $\theta_{m}, \dot{\theta}_{m}$ and $\ddot{\theta}_{m}$ are bounded.

Since all states associated with each joint i.e. $\theta, \dot{\theta}, \theta_{m}, \dot{\theta}_{m}$, and $I_{a}$ are bounded, vectors $\theta, \dot{\theta}, \theta_{m}, \dot{\theta}_{m}$ and $I_{a}$ are bounded. As a conclusion, based on the stability analysis, all required signals are bounded.

\section{Simulation}

The proposed type-2 PD fuzzy controller is simulated using an electrical flexible-joint articulated robot manipulator. The articulated manipulator is a serial link manipulator with three revolute joints as shown in Figure 4. The Denavit Hartenberg parameters of the articulated robot are given in Table 1, where the parameters $\theta_{i}, d_{i}, a_{i}$ and $\alpha_{i}$ are called the joint angle, link offset, link length and link twist, respectively. The dynamic parameters of manipulator are given in Table 2, where for the ith link, $m_{i}$ is the mass, $r_{c i}=\left[\begin{array}{lll}x_{c i} & y_{c i} z_{c i}\end{array}\right]^{\mathrm{T}}$ is the center of mass of the $i$ th frame and $I_{i}$ is the inertia tensor in the center of mass frame with the details as

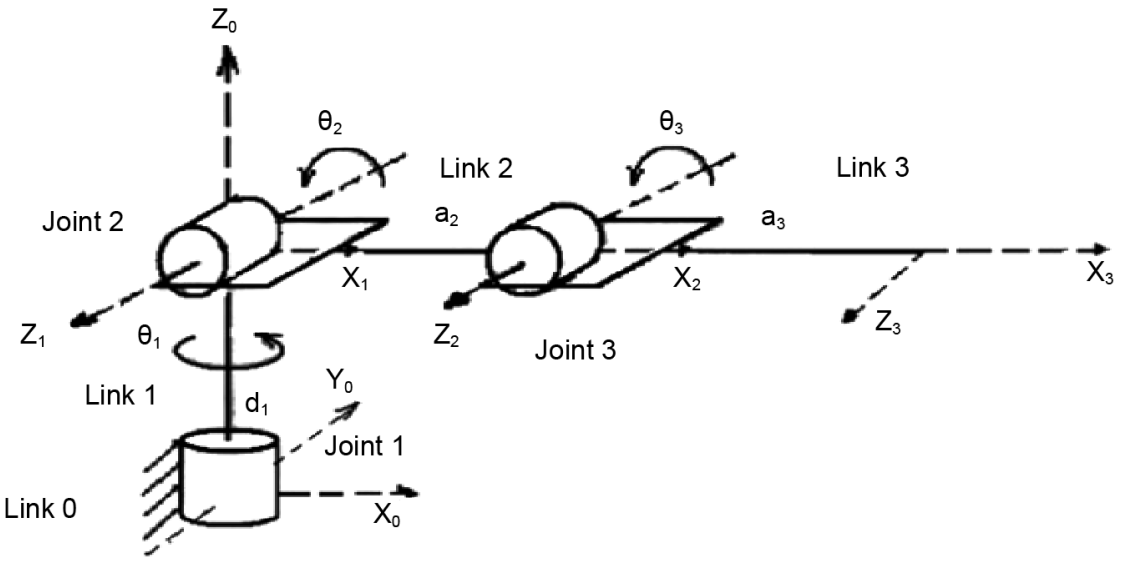

Figure 4. Symbolic representation of the articulated robot. 


$$
I_{i}=\left[\begin{array}{ccc}
I_{x x i} & I_{x y i} & I_{x z i} \\
I_{y x i} & I_{y y i} & I_{y z i} \\
I_{z x i} & I_{z y i} & I_{z z i}
\end{array}\right]
$$

The parameters of motors are given in Table 2. The desired joint trajectories are smooth as given by $\theta_{d}=1-\cos (0.05 \pi t)$. The maximum voltage of each motor is set to $u_{\max }=50 \mathrm{~V}$. To consider the parametric uncertainties, $\hat{k}_{b}$ and $\hat{r}$ are assumed to be $80 \%$ of their real values. Moreover, $k_{p_{1}}, k_{d_{1}}$ and $k_{o_{1}}$ are set to.01,200 and 30, $k_{p_{2}}, k_{d_{2}}$ and $k_{o_{2}}$ are set to 0.09, 400 and 200, $k_{p_{3}}, k_{d_{3}}$ and $k_{o_{3}}$ are set to $0.07,200$ and 100 , respectively.

Simulation 1: In This simulation, the proposed IT2PD controller is simulated. The performance of control system is shown in Figure 5 while the joint tracking error is reduced well. The external disturbance is zero by given $\varphi=0$ in system (14). The control system overcomes disturbances very well. The control efforts are shown in Figure 6. The motor voltages behave well under the maximum permitted value of $50 \mathrm{~V}$ as shown in Figure 6. In other words, both two figures indicate the fact that the tracking performance can be guaranteed. The simulation results confirm the effectiveness of the proposed method.

Table 1. The Denavit-Hartenberg parameters.

\begin{tabular}{ccccc}
\hline Link & $\theta$ & $d$ & $a$ & $\alpha$ \\
\hline 1 & $\theta_{1}$ & $d_{1}$ & 0 & $\pi / 2$ \\
2 & $\theta_{2}$ & 0 & $a_{2}$ & 0 \\
3 & $\theta_{3}$ & 0 & $a_{3}$ & 0 \\
\hline
\end{tabular}

Table 2. Motor parameters

\begin{tabular}{cccccccc}
\hline Motors & $\mathrm{R}$ & $\mathrm{k}_{\mathrm{b}}$ & $\mathrm{L}$ & $\mathrm{J}$ & $\mathrm{B}$ & $\mathrm{r}$ & $\mathrm{K}$ \\
\hline $1,2,3$ & 1.26 & 0.26 & 0.001 & 0.0002 & 0.001 & 0.02 & 500 \\
\hline
\end{tabular}

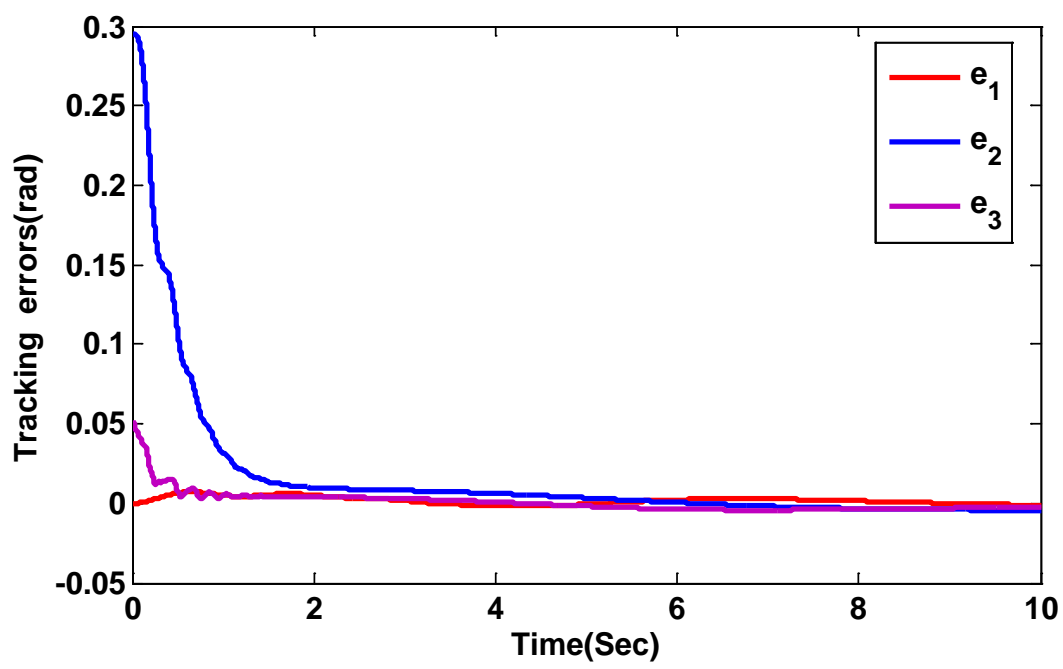

Figure 5. Performance of the interval type-2 fuzzy PD controller. 
Simulation 2: in this case a comparison between type-1 PD (T1PD) controller and T2PD controller is presented. In order to consider the robustness evaluation of the controllers, external disturbances are added to the robot system. The disturbance is inserted to the input of each motor as a periodic pulse function with a period of $2 \mathrm{~S}$, amplitude $\mp 4 \mathrm{~V}$, time delay of $0.7 \mathrm{~S}$, and pulse width $30 \%$ of period. This form of disturbance is an example of any form that can be applied but it includes jumps to cover the complex cases. To better assess the performance of both types of controllers in the face of external disturbance and unmodeled dynamics, the integral of squared errors (ISE) and the integral of absolute control input (ISU) are considered as a criterion

$$
\begin{aligned}
& \text { ISE }=\int_{0}^{10}\left(e_{1}^{2}+e_{2}^{2}+e_{3}^{2}\right) \mathrm{d} t \\
& \text { ISU }=\int_{0}^{10}\left(\left|u_{1}\right|+\left|u_{2}\right|+\left|u_{3}\right|\right) \mathrm{d} t
\end{aligned}
$$

where $e_{1}, e_{2}$ and $e_{3}$ are the tracking error of first, second and third link, respectively. In the meantime, $u_{1}, u_{2}$ and $u_{3}$ are the voltage of first, second and third motor, respectively.

The responses of control system using T1PD controller and IT2PD controller in the presence of disturbance are shown in Figure 7 and Figure 8, respectively. The tracking performance is shown in Figure 9 and it shows that the tracking error of T1PD controller is larger than the IT2PD controller. The ISU criterion for both controllers is shown in Figure 10, as well. From above simulation results, we obviously see that the T2PD controller can handle external disturbance very well. From Figure 9, the tracking performance of the IT2PD controller is much better than the tracking performance of the T1PD controller and in order to deal with external disturbance, as shown in Figure 10 the T1PD controller must spend more control effort. As a result, the simulation results confirm that IT2PD can outperform T1PD in controlling the flexible joint robot.

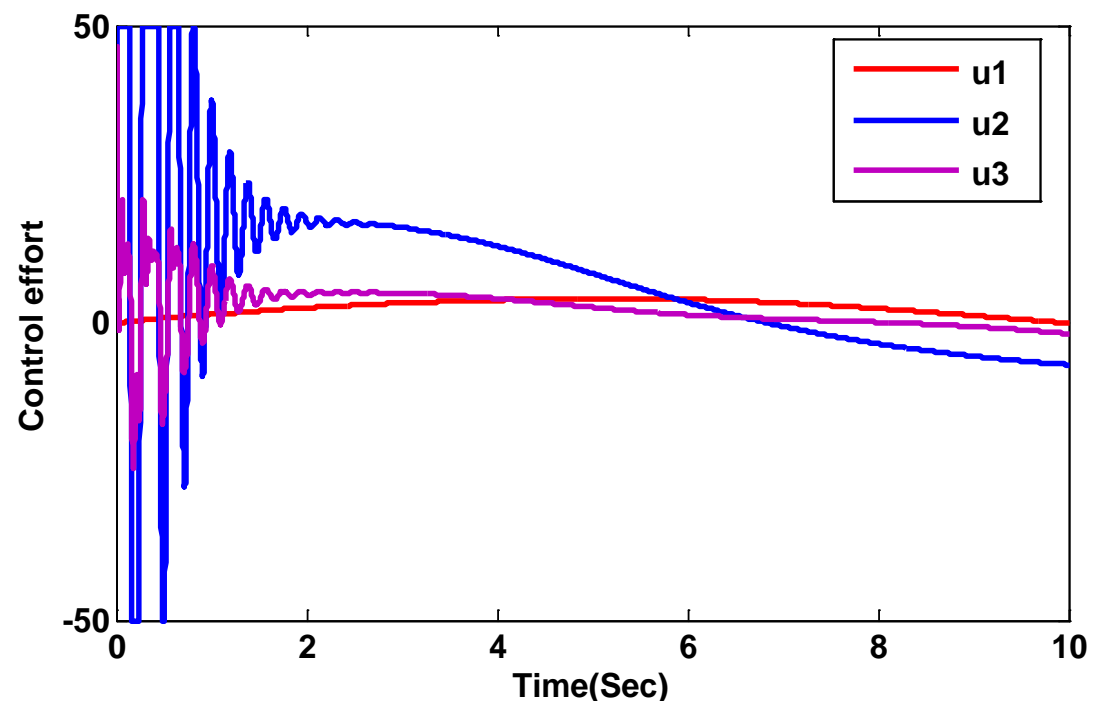

Figure 6. Control effort of the interval type-2 fuzzy PD controller. 


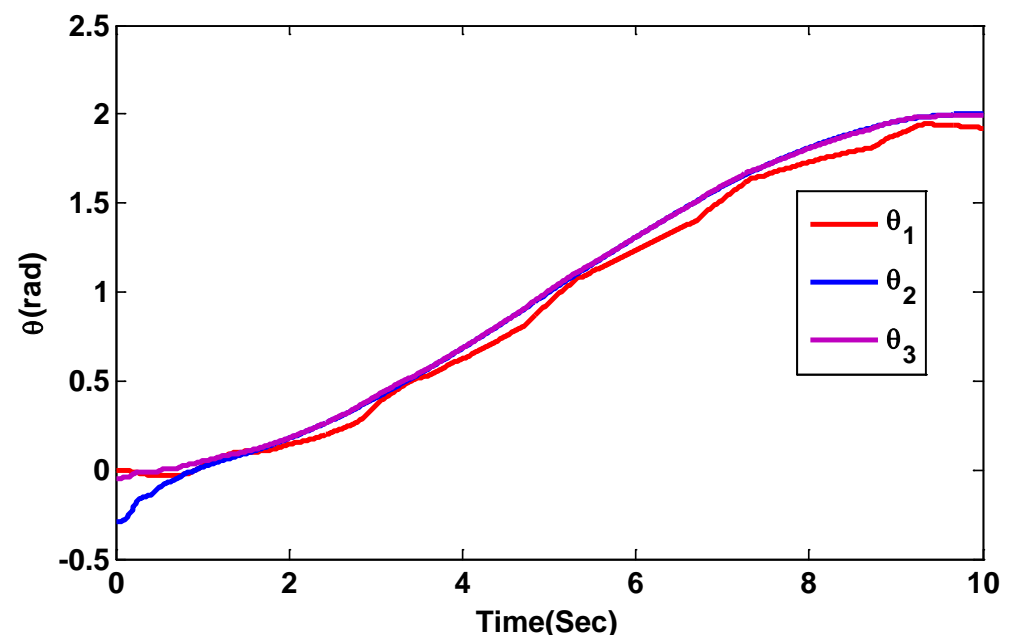

Figure 7. The response of control system using T1PD controller in the presence of noise.

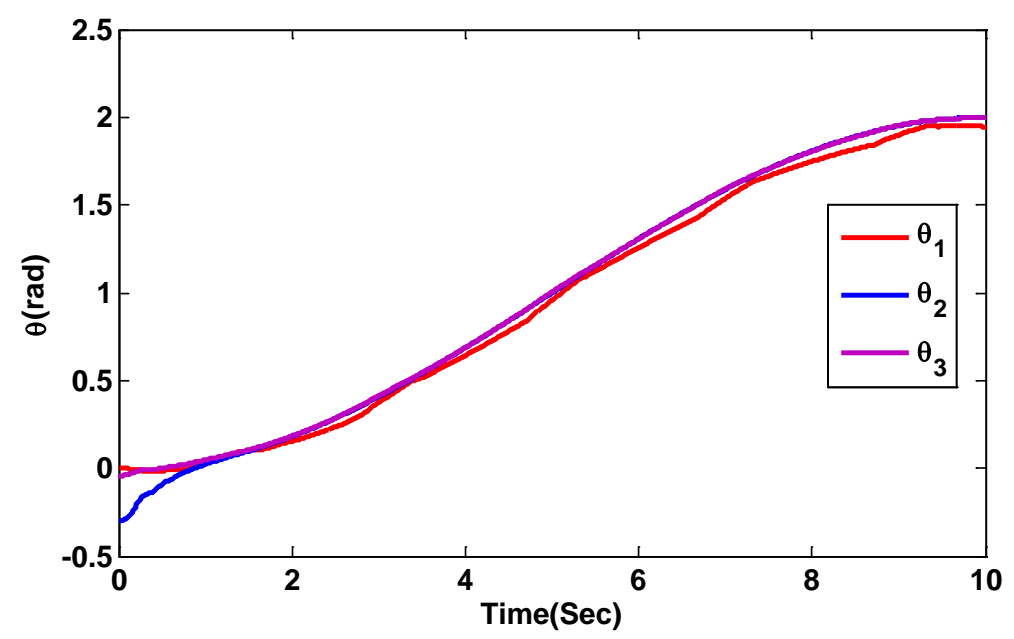

Figure 8. The response of control system using IT2PD controller in the presence of noise.

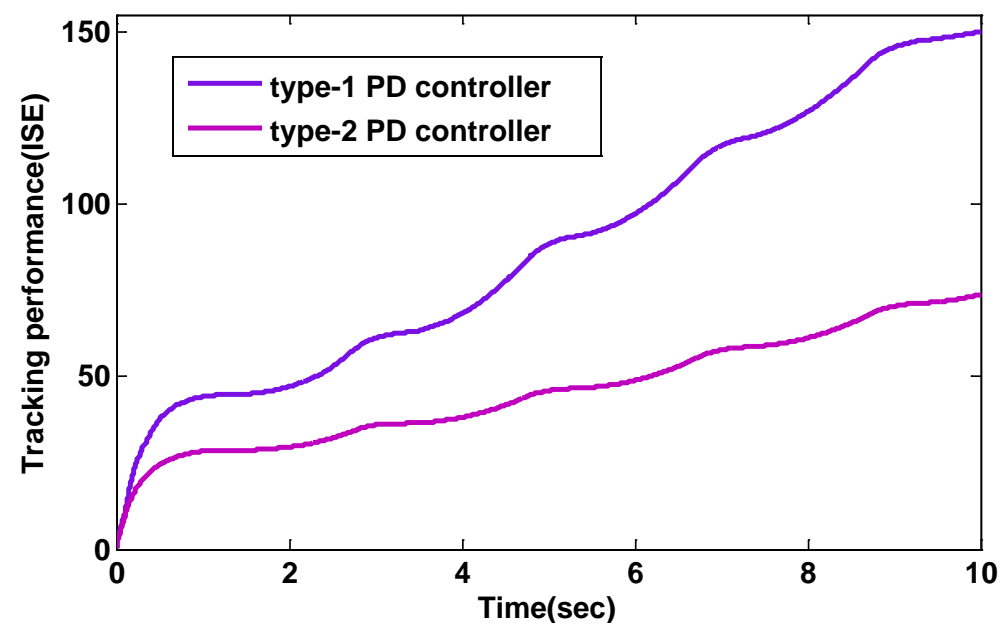

Figure 9. The tracking performance (ISE) of T1PD controller and T2PD controlle. 


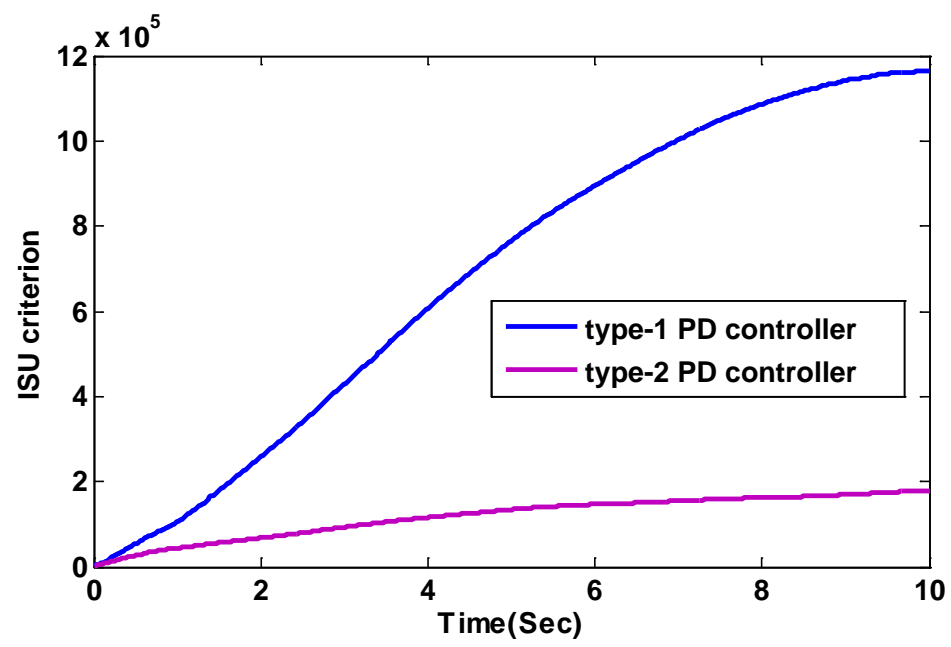

Figure 10. The ISU criterion for both controllers.

\section{Conclusion}

A novel interval type-2 fuzzy PD control was developed for tracking control of a flexible-joint robot using the voltage control strategy. The proposed method is free from manipulator dynamics and very simple in the form of a decentralized control. In addition, there are no restrictions on the joint stiffness gains. The stability analysis has verified the control method and the simulation results have confirmed its effectiveness. Compared to the previous controllers reported for the flexible-joint robots which use two control loops, it has a simpler structure using only one control loop. A comparison between interval type-2 fuzzy PD and type-1 fuzzy PD controller has been done and simulation results confirmed that type-2 fuzzy PD controller can handle external disturbance better than the type-1 fuzzy PD controller. In addition, it spends less control effort than the type-1 in order to deal with disturbance. Note that in the present paper a novel control approach has been proposed whereas in [29] type-2 fuzzy system has been directly used as a controller and Particle Swarm Optimization (PSO) used to optimize the control structure.

\section{Acknowledgements}

The authors gratefully appreciate the support of the Behbahan Khatam Alanbia University of Technology.

\section{References}

[1] Hagras, H.A. (2004) A Hierarchical Type-2 Fuzzy Logic Control Architecture for Autonomous Mobile Robots. IEEE Transactions on Fuzzy Systems, 12, 524-539. https://doi.org/10.1109/TFUZZ.2004.832538

[2] Hsiao, M.-Y., et al. (2008) Design of Interval Type-2 Fuzzy Sliding-Mode Controller. Information Sciences, 178, 1696-1716. https://doi.org/10.1016/j.ins.2007.10.019

[3] Mendel, J.M. (2001) Uncertain Rule-Based Fuzzy Logic System: Introduction and New Directions. Spinge, Berlin.

[4] Mendel, J.M. and John, R.B. (2002) Type-2 Fuzzy Sets Made Simple. Fuzzy Systems, 
IEEE Transactions on, 10, 117-127. https://doi.org/10.1109/91.995115

[5] Wu Woei Wan Tan, D. (2006) A Simplified Type-2 Fuzzy Logic Controller for Real-Time Control. ISA Transactions, 45, 503-516.

https://doi.org/10.1016/S0019-0578(07)60228-6

[6] Yager, R.R. (1980) Fuzzy Subsets of Type II in Decisions. Cybernetics and System, 10, 137-159. https://doi.org/10.1080/01969728008927629

[7] Lin, T.-C. and Roopaei, M. (2010) Based on Interval Type-2 Adaptive Fuzzy Ho Tracking Controller for SISO Time-Delay Nonlinear Systems. Communications in Nonlinear Science and Numerical Simulation, 15, 4065-4075.

https://doi.org/10.1016/j.cnsns.2010.01.029

[8] Khooban, M.H., Alfi, A. and Abadi, D.N.M. (2013) Control of a Class of Non-Linear Uncertain Chaotic Systems via an Optimal Type-2 Fuzzy Proportional Integral Derivative Controller. IET Science, Measurement \& Technology, 7, 50-58. https://doi.org/10.1049/iet-smt.2012.0092

[9] Dwivedy, S.K. and Eberhard, P. (2006) Dynamic Analysis of Flexible Manipulators, a Literature Review. Mechanism and Machine Theory, 41, 749-777. https://doi.org/10.1016/j.mechmachtheory.2006.01.014

[10] Tokhi, M.O. and Azad A.K. (2008) Flexible Robot Manipulators: Modelling, Simulation and Control. IET, Stevenage, 68. https://doi.org/10.1049/PBCE068E

[11] Book, W.J. (1979) Analysis of Massless Elastic Chains with Servo Controlled Joints. Journal of Dynamic Systems, Measurement, and Control, 101, 187-192. https://doi.org/10.1115/1.3426423

[12] Fateh, M.M. (2012) Nonlinear Control of Electrical Flexible-Joint Robots. Nonlinear Dynamics, 67, 2549-2559. https://doi.org/10.1007/s11071-011-0167-3

[13] Sweet, L.M. and Good, M. (1985) Redefinition of the Robot Motion-Control Problem. Control Systems Magazine, 5, 18-25. https://doi.org/10.1109/MCS.1985.1104955

[14] Kokotovic, P., Khali, H.K. and O'reilly, J. (1999) Singular Perturbation Methods in Control: Analysis and Design. SIAM, Vol. 25.

https://doi.org/10.1137/1.9781611971118

[15] De Luca, A., Isidori, A. and Nicolo, F. (1985) Control of Robot Arm with Elastic Joints via Nonlinear Dynamic Feedback. 24th IEEE Conference on Decision and Control.

[16] Chien, M.-C. and Huang, A.-C. (2007) Adaptive Control for Flexible-Joint Electrically Driven Robot with Time-Varying Uncertainties. IEEE Transactions on Industrial Electronics, 54, 1032-1038.

[17] Wilson, G. and Irwin, G. (1994) Robust Tracking of Elastic Joint Manipulators using Sliding Mode Control. Transactions of the Institute of Measurement and Control, 16, 99-107. https://doi.org/10.1177/014233129401600206

[18] Lih-Chang, L. and Chiang-Chuan, C. (1995) Rigid Model-Based Fuzzy Control of Flexible-Joint Manipulators. Journal of Intelligent and Robotic Systems, 13, 107-126. https://doi.org/10.1007/BF01254847

[19] Zeman, V., Patel, R. and Khorasani, K. (1997) Control of a Flexible-Joint Robot using Neural Networks. IEEE Transactions on Control Systems Technology, 5, 453-462. https://doi.org/10.1109/87.595927

[20] Li, Y., Tong, S. and Li, T. (2013) Adaptive Fuzzy Output Feedback Control for a Single-Link Flexible Robot Manipulator Driven DC Motor via Backstepping. Nonlinear Analysis: Real World Applications, 14, 483-494. 
[21] Tarn, T., et al. (1991) Effect of Motor Dynamics on Nonlinear Feedback Robot Arm Control. IEEE Transactions on Robotics and Automation, 7, 114-122.

[22] Fateh, M.M. (2012) Robust Control of Flexible-Joint Robots Using Voltage Control Strategy. Nonlinear Dynamics, 67, 1525-1537.

https://doi.org/10.1007/s11071-011-0086-3

[23] Spong, M.W. (1987) Modeling and Control of Elastic Joint Robots. Journal of Dynamic Systems, Measurement, and Control, 109, 310-318. https://doi.org/10.1115/1.3143860

[24] Fateh, M.M. (2008) On the Voltage-Based Control of Robot Manipulators. International Journal of Control, Automation, and Systems, 6, 702-712.

[25] Ailon, A., Lozano, R. and Gil, M. (2000) Iterative Regulation of an Electrically Driven Flexible-Joint Robot with Model Uncertainty. IEEE Transactions on Robotics and Automation, 16, 863-870. https://doi.org/10.1109/70.897798

[26] Liang, Q. and Mendel, J.M. (2000) Interval Type-2 Fuzzy Logic Systems: Theory and Design. IEEE Transactions on Fuzzy Systems, 8, 535-550.

[27] $\mathrm{Wu}, \mathrm{D}$. and Mendel, J.M. (2009) Enhanced Karnik-Mendel Algorithms. IEEE Transactions on Fuzzy Systems, 17, 923-934.

[28] Karnik, N.N. and Mendel, J.M. (2001) Centroid of a Type-2 Fuzzy Set. Information Sciences, 132, 195-220.

[29] Zirkohi, M.M., Fateh, M.M. and Shoorehdeli, M.A. (2013) Type-2 Fuzzy Control for a Flexible-Joint Robot using Voltage Control Strategy. International Journal of Automation and Computing, 10, 242-255. https://doi.org/10.1007/s11633-013-0717-x 